\title{
GEOMETRICAL ILLUSTRATION OF A THEOREM RELATING TO AN IRRATIONAL FUNCTION OF AN IMAGINARY VARIABLE.
}

[From the Proceedings of the London Mathematical Society, vol. viII. (1876-1877), pp. 212-214. Read May 11, 1876.]

IF we have $v$, a function of $u$, determined by an equation $f(u, v)=0$, then to any given imaginary value $x+i y$ of $u$ there belong two or more values, in general imaginary, $x^{\prime}+i y^{\prime}$ of $v$ : and for the complete understanding of the relation between the two imaginary variables, we require to know the series of values $x^{\prime}+i y^{\prime}$ which correspond to a given series of values $x+i y$, of $v, u$ respectively. We must for this purpose take $x, y$ as the coordinates of a point $P$ in a plane $\Pi$, and $x^{\prime}, y^{\prime}$ as the coordinates of a corresponding point $P^{\prime}$ in another plane $\Pi^{\prime}$. The series of values $x+i y$ of $u$ is then represented by means of a curve in the first plane, and the series of values $x^{\prime}+i y^{\prime}$ of $v$ by means of a corresponding curve in the second plane. The correspondence between the two points $P$ and $P^{\prime}$ is of course established by the two equations into which the given equation $f\left(x+i y, x^{\prime}+i y^{\prime}\right)=0$ breaks up, on the assumption that $x, y, x^{\prime}, y^{\prime}$ are all of them real. If we assume that the coefficients in the equation are real, then the two equations are

$$
\begin{aligned}
& f\left(x+i y, x^{\prime}+i y^{\prime}\right)+f\left(x-i y, x^{\prime}-i y^{\prime}\right)=0, \\
& f\left(x+i y, x^{\prime}+i y^{\prime}\right)-f\left(x-i y, x^{\prime}-i y^{\prime}\right)=0
\end{aligned}
$$

viz. if in these equations we regard either set of coordinates, say $(x, y)$, as constants, then the other set $\left(x^{\prime}, y^{\prime}\right)$ are the coordinates of any real point of intersection of the curves represented by these equations respectively.

I consider the particular case where the equation between $u, v$ is $u^{2}+v^{2}=a^{2}$ : we have here $(x+i y)^{2}+\left(x^{\prime}+i y^{\prime}\right)^{2}=a^{2}$ : so that, to a given point $P$ in the first plane, there

C. IX. 
correspond in general two points $P_{1}^{\prime}, P_{2}^{\prime}$ in the second plane: but to each of the points $A$ and $B$, coordinates $(a, 0)$ and $(-a, 0)$, there corresponds only a single point in the second plane.

We have here a particular case of a well-known theorem: viz. if from a given point $P$ we pass by a closed curve, not containing within it either of the points $A$ or $B$, back to the initial point $P$, we pass in the other plane from $P_{1}^{\prime}$ by a closed curve back to $P_{1}^{\prime}$; and similarly from $P_{2}^{\prime}$ by a closed curve back to $P_{2}^{\prime}$ : but if the closed curve described by $P$ contain within it $A$ or $B$, then, in the other plane, we pass continuously from $P_{1}^{\prime}$ to $P_{2}^{\prime}$; and also continuously from $P_{2}^{\prime}$ to $P_{1}^{\prime}$.

The relations between $(x, y),\left(x^{\prime}, y^{\prime}\right)$ are

$$
\begin{aligned}
x^{\prime 2}-y^{\prime 2} & =a^{2}-\left(x^{2}-y^{2}\right), \\
x^{\prime} y^{\prime} & =-x y,
\end{aligned}
$$

whence also

$$
\left(x^{\prime 2}+y^{\prime 2}\right)^{2}=a^{4}-2 a^{2}\left(x^{2}-y^{2}\right)+\left(x^{2}+y^{2}\right)^{2} .
$$

And if the point $(x, y)$ describe a curve $x^{2}+y^{2}=\phi\left(x^{2}-y^{2}\right)$, then will the point $\left(x^{\prime}, y^{\prime}\right)$ describe a curve $x^{\prime 2}+y^{\prime 2}=\psi\left(x^{\prime 2}-y^{\prime 2}\right)$, obtained by the elimination of $x^{2}-y^{2}$ from the two equations

$$
\begin{aligned}
x^{\prime 2}-y^{\prime 2} & =a^{2}-\left(x^{2}-y^{2}\right) \\
\left(x^{\prime 2}+y^{\prime 2}\right)^{2} & =a^{4}-2 a^{2}\left(x^{2}-y^{2}\right)+\phi\left(x^{2}-y^{2}\right)
\end{aligned}
$$

viz. this is

$$
\left(x^{\prime 2}+y^{\prime 2}\right)^{2}=-a^{4}+2 a^{2}\left(x^{\prime 2}-y^{\prime 2}\right)+\phi\left\{a^{2}-\left(x^{\prime 2}-y^{\prime 2}\right)\right\} \text {. }
$$

In particular, if the one curve be $\left(x^{2}+y^{2}\right)^{2}=\alpha+\beta\left(x^{2}-y^{2}\right)$; then the other curve is

that is,

$$
\left(x^{\prime 2}+y^{\prime 2}\right)^{2}=-a^{4}+2 a^{2}\left(x^{\prime 2}-y^{\prime 2}\right)+\alpha+\beta\left\{\alpha^{2}-\left(x^{\prime 2}-y^{\prime 2}\right)\right\},
$$

where

$$
\left(x^{\prime 2}+y^{\prime 2}\right)^{2}=\alpha^{\prime}+\beta^{\prime}\left(x^{\prime 2}-y^{\prime 2}\right)
$$

$$
\alpha^{\prime}=-a^{4}+\beta a^{2}+\alpha, \quad \beta^{\prime}=2 a^{2}-\beta .
$$

Writing for greater simplicity $a=1$, then $\alpha^{\prime}=-1+\alpha+\beta, \beta^{\prime}=2-\beta$; in particular, if $\alpha=0$, then $\alpha^{\prime}=-1+\beta, \beta^{\prime}=2-\beta$.

Supposing successively $\beta<1, \beta=1$, and $\beta>1$, then in each case $P$ describes a closed curve or half figure-of-eight, as shown in the annexed $P$-figure; but in the first case the point $A$ is inside the curve, in the second case on it, and in the third case outside it, as shown by the letters $A, A, A$ of the figure; and, corresponding to the three cases respectively, we have the three $P^{\prime}$-figures, the curve in the first of them consisting of two ovals, in the second of them being a figure of eight, and in the third a twice-indented or pinched oval: the small figures $1,2,3,4$ in the $P$-figure, and $1,2,3,4$ and $1^{\prime}, 2^{\prime}, 3^{\prime}, 4^{\prime}$ in the $P^{\prime}$-figures serve to show the corresponding 
positions of the points $P$ and $P_{1}^{\prime}, P_{2}^{\prime}$ respectively; and the courses are further indicated by the arrows. And we thus see how the two separate closed curves described

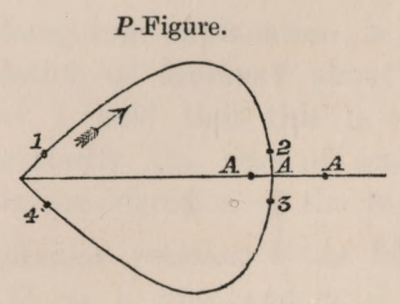

$P^{\prime}$-Fig. 2.

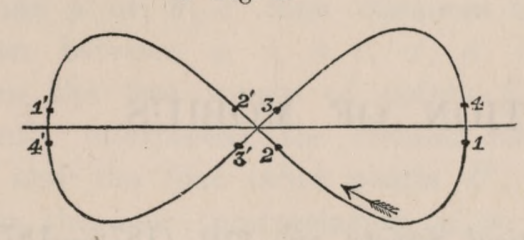

$P^{\prime}$-Fig. 1.

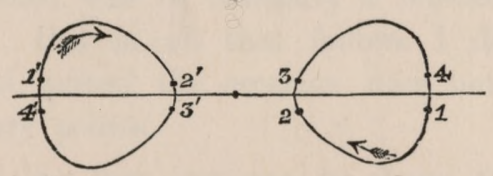

$P^{\prime}$-Fig. 3.

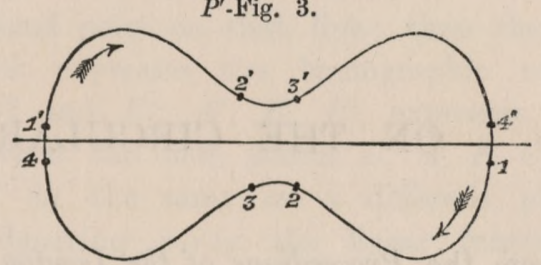

by $P_{1}^{\prime}$ and $P_{2}^{\prime}$, as in figure 1 , change into the single closed curve described one half of it by $P_{1}^{\prime}$ and the other half of it by $P_{2}^{\prime}$ as in figure 3 . 